University of Nebraska - Lincoln

DigitalCommons@University of Nebraska - Lincoln

February 2003

\title{
Life-History Evolution and the Microevolution of Intermediary Metabolism: Activities of Lipid-Metabolizing Enzymes in Life- History Morphs of a Wing-Dimorphic Cricket
}

Anthony J. Zera

University of Nebraska - Lincoln, azera1@unl.edu

Zhangwu Zhao

University of Nebraska - Lincoln, zhaozw_00@yahoo.com

Follow this and additional works at: https://digitalcommons.unl.edu/bioscizera

Part of the Microbiology Commons

Zera, Anthony J. and Zhao, Zhangwu, "Life-History Evolution and the Microevolution of Intermediary Metabolism: Activities of Lipid-Metabolizing Enzymes in Life-History Morphs of a Wing-Dimorphic Cricket" (2003). Anthony Zera Publications. 9.

https://digitalcommons.unl.edu/bioscizera/9

This Article is brought to you for free and open access by the Papers in the Biological Sciences at DigitalCommons@University of Nebraska - Lincoln. It has been accepted for inclusion in Anthony Zera Publications by an authorized administrator of DigitalCommons@University of Nebraska - Lincoln. 


\title{
Life-History Evolution and the Microevolution of Intermediary Metabolism: Activities of Lipid-Metabolizing Enzymes in Life-History Morphs of a Wing-Dimorphic Cricket
}

\author{
Anthony J. Zera and Zhangwu Zhao \\ School of Biological Sciences, University of Nebraska, Lincoln, Nebraska 68588-0118 \\ E-mail: azera1@unlnotes.unl.edu; zhaozw_00@yahoo.com
}

\begin{abstract}
Although a considerable amount of information is available on the ecology, genetics, and physiology of life-history traits, much more limited data are available on the biochemical and genetic correlates of life-history variation within species. Specific activities of five enzymes of lipid biosynthesis and two enzymes of amino acid catabolism were compared among lines selected for flight-capable (LW[f]) versus flightless (SW) morphs of the cricket Gryllus firmus. These morphs, which exist in natural populations, differ genetically in ovarian growth (100-400\% higher in SW) and aspects of flight capability including the size of wings and flight muscles, and the concentration of triglyceride flight fuel (40\% greater in LW[f]). Consistently higher activity of each enzyme in LW(f) versus SWselected lines, and strong co-segregation between morph and enzyme activity, demonstrated genetically based covariance between wing morph and enzyme activity. Developmental profiles of enzyme activities strongly paralleled profiles of triglyceride accumulation during adulthood and previous measures of in vivo lipid biosynthesis. These data strongly imply that genetically based elevation in activities of lipogenic enzymes, and enzymes controlling the conversion of amino acids into lipids, is an important cause underlying the elevated accumulation of triglyceride in the LW(f) morph, a key biochemical component of the trade-off between elevated early fecundity and flight capability. Global changes in lipid and amino-acid metabolism appear to have resulted from microevolutionary alteration of regulators of metabolism. Finally, strong genotype $\times$ environment (diet) interactions were observed for most enzyme activities. Future progress in understanding the functional causes of life-history evolution requires a more detailed synthesis of the fields of life-history evolution and metabolic biochemistry. Wing polymorphism is a powerful experimental model in such integrative studies.
\end{abstract}

Keywords: Adaptation, enzymes, intermediary metabolism, life history, trade-off, wing polymorphism

The functional causes of life-history evolution have been a central focus of life-history research for over six decades (Fisher 1930; Pianka 1981; Townsend and Calow 1981; Service 1987; Mole and Zera 1993; Djawdan et al. 1996; Zera et al. 1998; Zera and Harshman 2001). At issue are the physiological, biochemical, and molecular processes that have been altered by natural selection to produce modified life-history traits (e.g., enhanced early reproduction) and trade-offs between traits (e.g., the coupling of decreased longevity with enhanced early reproduction). An increasing number of studies have identified physiological (e.g., energetic and endocrine) correlates of life-history variation and trade-offs within species (Service 1987; Chippindale et al. 1996; Djawdan et al. 1996; Harshman and Schmidt 1998; Zera and Huang 1999; Harshman and Hoffmann 2000; Zera and Cisper 2001; Zera and Harshman 2001; Zera and Larsen 2001). However, much less is known about the biochemical underpinnings of life-history variation or trade-offs.

A priori, evolutionary modification of intermediary metabolism is expected to be an important factor in life-history evolution. For example, the evolution of a life history characterized by increased early age fecundity must involve modifications of protein metabolism related to egg yolk protein biosynthesis. Similarly, life histories that involve increased longevity or resistance to stressful conditions (e.g., tolerance to desiccation or starvation) are typically associated with elevated accumulation of energy reserves, most notably triglyceride or glycogen (Zera and Harshman 2001). The evolution of these life-history traits must involve dramatic modifications in carbohydrate and lipid metabolism. Finally, because of strong interactions among metabolic pathways, evolutionary modification of a particular pathway of metabolism is expected to result in alterations in other pathways. Despite the potential importance of microevolutionary modification of intermediary metabolism in life-history evolution, only limited information is available on the specific metabolic pathways, enzymes, or regulatory controls that have been altered in genotypes, populations, or species with different life histories. This paucity of information constitutes a major roadblock to understanding the mechanistic basis of life-history evolution.

The present study is part of a series whose overall goal is to identify, in detail, the physiological, biochemical, and molecular causes of genetically based life-history variation and trade-offs in the wing polymorphic cricket Gryllus firmus. This species consists of discrete morphs, found within the same population, which differ dramatically in life-history traits relating to dispersal and reproduction (Veazy et al. 1976; reviewed in Zera and Denno 1997; Zera and Harshman 2001). One morph has a fully developed flight apparatus including long wings, large flight muscles, and large stores of triglyceride flight fuel. An alternate flightless morph has 
reduced wings and flight muscles, reduced triglyceride stores, but substantially larger ovaries and elevated fecundity during the first week of adulthood (Zera et al. 1997, 1998; Zera and Brink 2000; Zera and Bottsford 2001; Zera and Cisper 2001; Zera and Larsen 2001). Thus, in G. firmus, a dramatic trade-off exists between early-age reproduction and flight capability. Importantly, artificial selection experiments have demonstrated a genetic basis for differences between the morphs in each of the morphological, physiological, and reproductive traits mentioned above (see above references and Materials and Methods).

Our focus in the present study is evolutionary modification of lipid metabolism resulting in specialization of G. firmus morphs for enhanced early reproduction versus dispersal capability. Lipid (e.g., triglyceride) is the major flight fuel in many insects including species of Gryllus (Zera et al. 1999). During the first week of adulthood, the flight-capable (LW[f]) morph of G. firmus accumulates a substantially greater amount of triglyceride than the flightless SW morph (Zera and Larsen 2001). During the same period ovarian growth is substantially $(50-75 \%)$ reduced in the LW(f) morph. Although these results suggest a biochemically based trade-off between nutrients allocated to pathways of lipid versus protein biosynthesis, little is known about the specific aspects of lipid metabolism that have been altered during the evolution of the high-fecundity SW morph. In the present study we compared the activities of a diverse set of enzymes involved in the biosynthesis of lipid and the conversion of amino acids into lipid in G. firmus. Because we are primarily interested in the microevolution of intermediary metabolism and life histories, comparisons were made between morphs from artificially selected lines that differ genetically in the concentration of lipid and triglyceride, aspects of dispersal capability (size of wings and flight muscles), and early-age reproduction (ovarian growth).

\section{Materials and Methods}

\section{Background on Gryllus firmus: Morphs, Selected Stocks, and Rearing Conditions}

Gryllus firmus, the sand cricket, exists in the southeastern United States as a long-winged (LW) morph, some of which are capable of flight, or as a short-winged (SW) morph that is obligatory flightless (Veazy et al. 1976; Zera et al. 1997). Except in a few rare cases, all SW females molt into adults with white, nonfunctional, underdeveloped flight muscles. All LW females initially have fully developed, functional (pink) flight muscles at or shortly after the adult molt. Individuals with functional flight muscles are denoted as LW(f). Upon the onset of ovarian growth, some LW(f) individuals histolyze (degenerate) their flight muscles, thus becoming flightless (denoted LW[h]; see below and Zera et al. 1997). Very few LW(h) individuals are currently produced in the selected lines during the first week of adulthood ( $<5 \%$ of all crickets), and it is uncertain whether the $\mathrm{LW}(\mathrm{h})$ morph is produced due to variation in genetic or environmental factors. In the present study we were primarily interested in biochemical differences between genetically specified morphs, and thus we did not measure enzyme activities in the LW(h) morph (phenotypic characterization of enzyme activities of the LW[h] morph can be found in Zhao and Zera 2001).

The G. firmus females used in the present study were taken from lines artificially selected for wing morph. The experimen- tal design consisted of three blocks (independent selection trials), each of which contained a line selected for the LW morph (L line), a line selected for the SW morph (S line), and a control (C) line (see fig. 1 of Zera and Bottsford 2001; Zera and Cisper 2001). The number following the line type (L or S) designates the block from which it is derived. Only selected (L and S) lines are considered here, which are close to being pure breeding (i.e., L lines produce $>90 \%$ LW females, whereas S lines produce $>90 \% \mathrm{SW}$ females). All lines were raised under the same environmental conditions. Briefly, crickets were reared at $28^{\circ} \mathrm{C}$ under a 16:8 L:D photoperiod, at a density of 80 and 40 crickets per 10-gallon aquarium during the penultimate and last stadium, respectively. Crickets were switched from the standard dry diet to the standard $100 \%$ wet diet (same components as the dry diet but made up in 2.5\% aqueous agar; see Zera and Larsen 2001) during the penultimate stadium. Rearing crickets on the dry diet during most of the juvenile stage substantially minimizes the labor involved in feeding, but increases the expression of the SW morph in LW-selected lines. Switching crickets to the wet diet starting at the penultimate stage reduces the expression of the SW morph in LW-selected lines, while not increasing the expression of the LW(f) morph in SW-selected lines, thus resulting in genetic stocks that are nearly pure breeding (i.e., they produce $>90 \%$ of the selected morph). Crickets used for experiments were checked for molting at 24-h intervals and equal numbers of newly molted females and males were raised together at a density of four to six per 1-gallon plastic box or eight to 12 per 3-gallon plastic box. Because this study focused exclusively on the trade-off between lipid metabolism and ovarian growth, enzyme activities were only measured in females.

No oviposition material was provided, so that females would retain all of their eggs and hence ovarian masses could be quantified to estimate morph-specific reproductive effort. There is virtually no oviposition during the period of adult development studied (first 5 days of adulthood), even when oviposition material is provided (for a more detailed discussion see materials and methods of Zhao and Zera 2001). Additional information on the origin of the lines of G. firmus and breeding can be found in Zera and Huang (1999), Zera and Bottsford (2001), and Zera and Cisper (2001). In the present study, crickets were used during generation 18 of selection. Previous studies have documented that LW(f) individuals from the $\mathrm{L}$ lines and $\mathrm{SW}$ individuals from the $\mathrm{S}$ lines differ genetically in the size of wings, flight muscles, ovaries, and in the amount of total lipid and triglyceride (Zera and Bottsford 2001; Zera and Cisper 2001; Zera and Larsen 2001). For example, whole-body total lipid and triglyceride contents (mass-adjusted by ANCOVA) were significantly higher in the LW-selected versus the SW-selected lines of each of three independent selection trials (blocks), whereas grand mean total lipid and triglyceride contents were significantly higher in LW-selected versus SWselected lines across the three blocks (Zera and Larsen 2001).

Enzyme-specific activities were quantified on day 0 (day of molt) and day 5 of adulthood. Day 5 was chosen because this is the period of time during which there is a dramatic trade-off between ovarian growth and accumulation of triglyceride reserves in the LW(f) and SW morphs (see Zera and Larsen 2001; see Discussion). Day 0 was chosen as a baseline, to determine enzyme activities prior to the occurrence of the trade-off mentioned above. 
To determine the extent to which differences between morphs in enzyme activities were contingent upon diet, comparisons were made between morphs fed one of three diets during adulthood. These are the same diets that were used in our prior study of lipid reserves in morphs of G. firmus (Zera and Larsen 2001). All juveniles were raised on the standard diet (termed "high"), which had been used in earlier feeding studies (Mole and Zera 1993; Zera et al. 1998; Zera and Brink 2000). All individuals were fed this diet up to and including the penultimate and last juvenile stadia. Starting on the day of adult molt, one group of adults was fed this same diet. A second group of adults was fed a low nutrient diet (termed "low" diet) in which $75 \%$ of the dry mass of the high-nutrient diet had been replaced with nonnutritive cellulose. Growth in two Gryllus species, G. assimilis and G. firmus, is substantially reduced on this diet (Zera et al. 1998; T. Rooneem and A. J. Zera, unpublished data). A third group of crickets was fed a high-carbohydrate diet starting on the first day of adulthood. In this diet $67 \%$ of the cellulose of the low-nutrient diet was replaced with sucrose (termed "sucrose" diet; $~ 9 \%$ sucrose w/w). A high-carbohydrate diet was used because many studies have shown that activities of lipogenic enzymes are strongly affected by the quantity of carbohydrate in the diet (Geer and Laurie-Ahlberg 1984; Downer 1985). All diets had the same dry mass and water content.

\section{Enzyme Assays}

Specific activities of seven enzymes involved in lipid and amino acid metabolism were quantified in homogenates of fat body, the major organ of lipid metabolism in insects (Fig. 1). The fol- lowing enzymes were assayed: (1) $\mathrm{NADP}^{+}$-isocitrate dehydrogenase $\left(\mathrm{Nadp}^{+}-\mathrm{Idh}\right.$; E.C. 1.1.1.42); (2) glucose-6-phosphate dehydrogenase (G-6-pdh; E.C. 1.1.1.49); (3) malic enzyme (Me; E.C. 1.1.1.40); (4) fatty acid synthase ( $\mathrm{Fas})$; (5) ATP-citrate lyase ( $\mathrm{Acl}$; E.C. 4.1.3.8); (6) alanine aminotransferase (AlaAT; E.C. 2.6.1.2); and (7) aspartate aminotransferase (AspAT; E.C. 2.6.1.1). Soluble protein was determined using the bicinchoninic acid assay using bovine serum albumen (fraction V) as the standard (Stoscheck 1990). Specific assay protocols (with the exception of aspartate aminotransferase) and methods of homogenization are described in Zhao and Zera (2001). Aspartate aminotransferase was assayed as follows: $50 \mathrm{mM}$ aspartate, $0.11 \mathrm{mM}$ pyridoxyl-5-phosphate, $15 \mathrm{mM}$ alpha-ketoglutarate, $0.18 \mathrm{mM}$ NADH, 0.7 units of malate dehydrogenase in $100 \mathrm{mM}$ MOPS buffer ( $\mathrm{pH}$ 7.3).

\section{Fat Body and Ovarian Mass}

Total fat body content of the thorax and abdomen was estimated in G. firmus morphs on days 0 and 5 of adulthood. Crickets were weighed and all fat bodies in the thorax and abdomen were dissected out, blotted dry, and weighed. Fat bodies from the legs and head were not removed because most are located in the thorax and abdomen and they are very difficult to remove from the head or legs. Both ovaries were also removed from LW(f) and SW females on day 5 of adulthood and weighed.

\section{Relative Total-Fat-Body Enzyme Activity}

Morph-specific differences in lipid biosynthetic capacity could result from differences in either or both of the following factors:

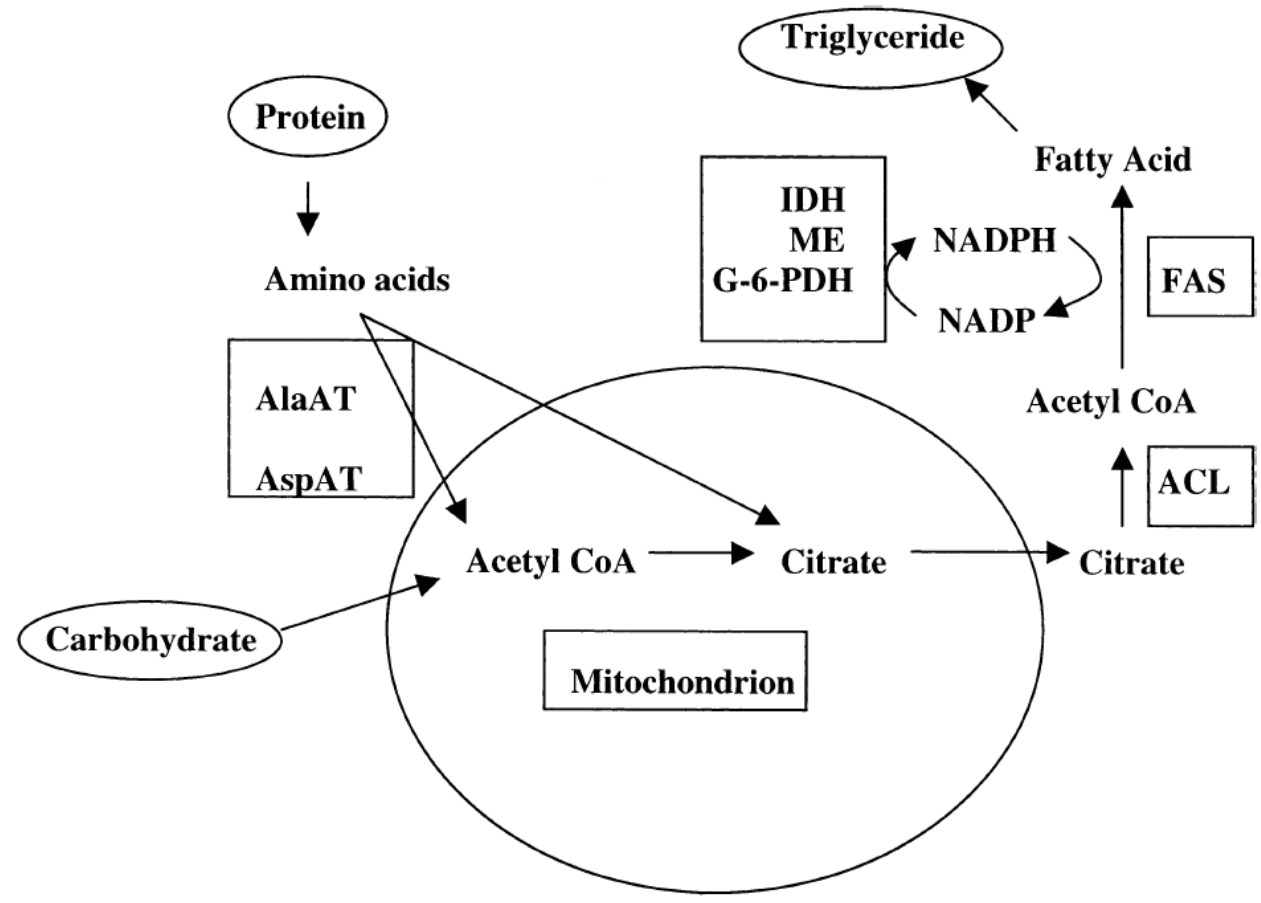

Figure 1. Pathways of lipid biosynthesis and enzymes involved (enclosed in boxes). See Materials and Methods for full names of enzymes and Discussion for description of pathways. NADPH and NADP refer, respectively, to reduced and oxidized forms of nicotinamide adenine dinucleotide phosphate. 
Table 1. Fat body mass, ovarian mass, and whole body mass, in flight-capable (LW[f]) and flightless (SW) Gryllus firmus from three pairs of lines (three blocks) selected for the LW or SW phenotype and raised on three diets. Values are means ( \pm SEM) based on six to eight females for each sample except for block 2, day 5 , LW, low diet $(N=4)$ and block 1 , day $0, \mathrm{LW}(N=5)$. Asterisks following means indicate differences between morphs within a block at $* P<0.05 ; * * P<0.01$; $* * * P<0.005$. Asterisks following results of paired $t$-tests indicate differences between grand means of morphs across blocks (i.e., genetic differences).

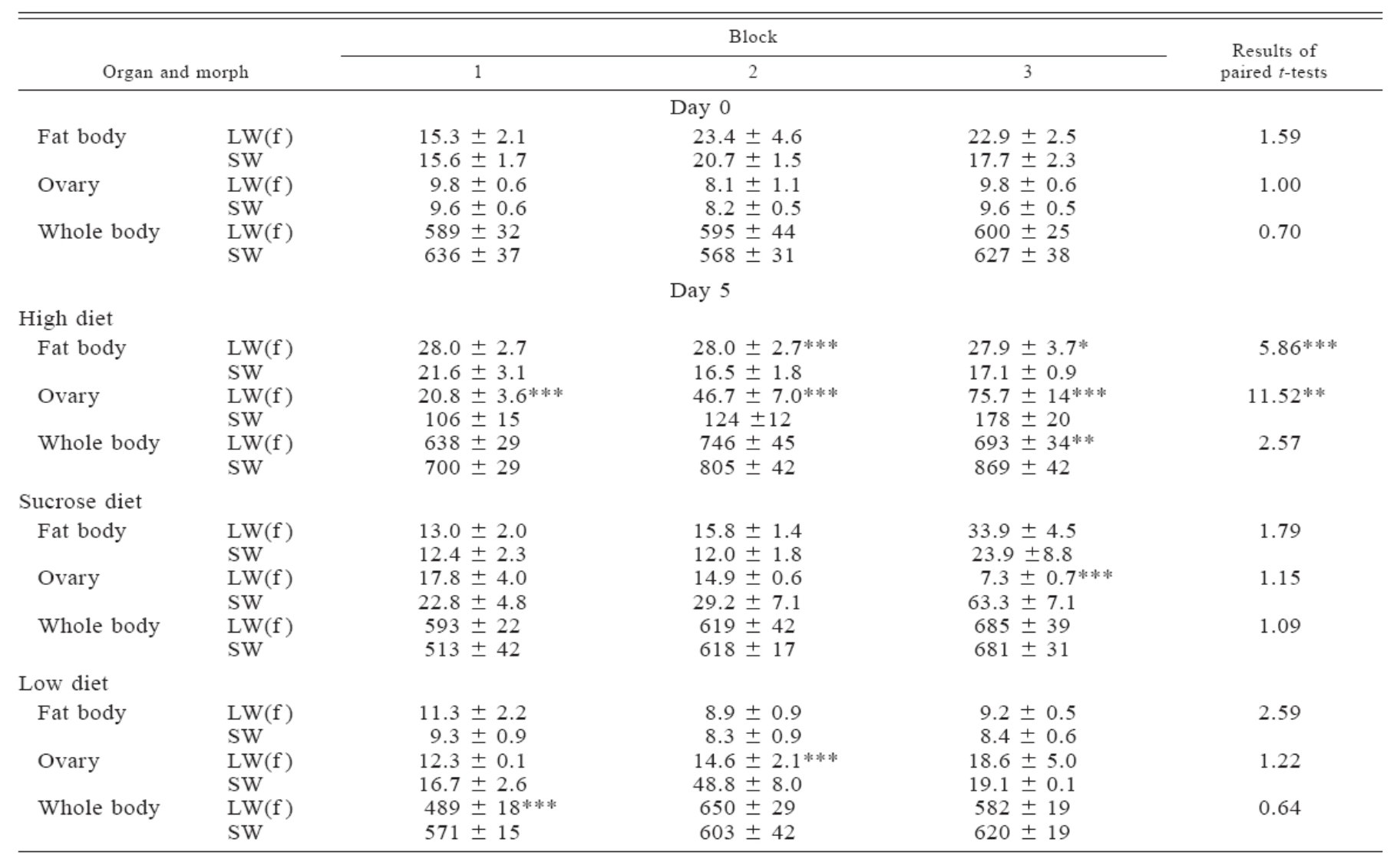

specific activity of biosynthetic enzymes (activity standardized to protein content of fat body) or mass of the fat body, the main organ of lipid biosynthesis. Measurement of total fat body mass is very time consuming and could not be done for individuals whose enzyme activities were measured. Furthermore, the large number of morphs, diets, and blocks precluded estimating fat body mass in individuals via regression. We therefore measured relative total-fat-body enzyme activity for morphs in the following way: Enzyme-specific activity of an individual morph raised on a particular diet was multiplied by mean fat body mass for that morph and/or diet obtained from a separate group of crickets (described above). Protein content per unit mass of fat body did not differ between morphs on a particular diet (ANOVA; $P>0.1$ ). Thus, the product of specific activity (i.e., activity per microgram fat body protein) and total fat body mass is proportional to total fat body activity (=activity per unit fat body mass $\times$ total fat body mass).

\section{Line Crosses}

To further establish the genetic basis of enzyme activity differences between morphs and to determine the degree of cosegregation (covariance, correlation) between enzyme activity and various morphological and reproductive aspects of wing morph, single- pair crosses were performed between the L-1 and S-1 lines. An $\mathrm{F}_{1} \mathrm{SW}$ female derived from one of these crosses was backcrossed to a LW male of the L-1 line (cross 9), and another SW $F_{1}$ female was backcrossed to a SW male of the S-1 line (cross 10). Activities of six enzymes (Fas, Acl, G-6-pdh, Idh, Me, AspAT), ovarian mass, wing length, and flight muscle phenotype were measured in LW and $\mathrm{SW} \mathrm{F}_{2}$ progeny of crosses 9 and 10.

\section{Statistical Analyses}

The main goal of the present study was to determine if activities of lipid-metabolizing enzymes differ genetically between morphs from lines selected for the LW(f) or SW morph. This was done in two ways. First, morph means were compared across the three blocks by paired $t$-tests (as performed previously in Zera and Bottsford 2001; Zera and Cisper 2001; Zera and Larsen 2001; see also Rose et al. 1996). This test essentially determines whether there is a consistent difference between the mean specific activity of an enzyme in LW(f) females of an L line versus SW females from the $\mathrm{S}$ line of the same block, averaged across the three blocks. Such consistent differences are indicative of genetically based variation in enzyme activities (see above references for additional details). The paired $t$-test preformed in this manner 
Table 2. Specific activities of fat body enzymes on day 0 (day of molt to adulthood) in flight-capable (LW[f]) and flightless (SW) Gryllus firmus. Values are mean specific activity (nmol/min/mg protein) followed by SEM and sample size. All paired $t$-tests of means were nonsignificant $(P>0.1)$; activities that differed between LW(f) and SW morphs within a block are denoted by asterisks $(* P<0.05, * * * P<0.005)$.

\begin{tabular}{|c|c|c|c|c|}
\hline & & \multicolumn{3}{|c|}{ Block } \\
\hline \multicolumn{2}{|c|}{ Enzyme and morph } & 1 & 2 & 3 \\
\hline Fas & LW(f) & $3.6 \pm 0.47(8)^{* * * *}$ & $4.8 \pm 0.21(7)$ & $4.8 \pm 1.08(6)$ \\
\hline $\mathrm{ACl}$ & SW & $6.0 \pm 0.77(8)$ & $9.2 \pm 1.44(7)$ & $17.4 \pm 4.8(5)$ \\
\hline \multirow{2}{*}{$G-6-p d h$} & $\mathrm{LW}(\mathrm{f})$ & $28.2 \pm 2.7(10)^{*}$ & $42.8 \pm 4.4(5)$ & $69.6 \pm 14.6(8)$ \\
\hline & SW & $20.7 \pm 1.1(8)$ & $41.1 \pm 2.2(5)$ & $60.7 \pm 16.3(7)$ \\
\hline \multirow[t]{2}{*}{$\mathrm{Me}$} & LW(f) & $4.7 \pm 0.67(5)$ & $6.4 \pm 0.70(5)$ & $12.0 \pm 2.8(8)$ \\
\hline & SW & $3.9 \pm 0.75$ & $7.1 \pm 0.51$ & $13.4 \pm 2.9(8)$ \\
\hline \multirow[t]{2}{*}{$A \operatorname{sp} A T$} & LW(f) & $116 \pm 12(5)$ & $101 \pm 2.6(5)$ & $55.3 \pm 5.7(7)$ \\
\hline & SW & $129 \pm 31(5)$ & $104 \pm 5.7(5)$ & $46.9 \pm 5.6(7)$ \\
\hline \multirow[t]{2}{*}{ AlaAT } & LW(f) & $2.9 \pm 0.17(5)$ & $3.1 \pm 0.58(5)$ & $5.7 \pm 1.2(5)$ \\
\hline & SW & $3.3 \pm 0.44(5)$ & $3.1 \pm 0.38(5)$ & $6.0 \pm 0.78(6)$ \\
\hline
\end{tabular}

is conservative because it tests for genetic differences of equivalent magnitude between LW(f) and SW morphs in the various blocks. For example, it is conceivable that morphs might differ in enzyme activities due to genetic causes, but that the magnitude of the morph differences might be heterogeneous across blocks due to the action of block-specific alleles that differentially affect enzyme activity. Thus, genetic differences between LW(f) and SW females in enzyme activities were also assessed by simply inspecting the consistency of differences between LW(f) and SW females measured within a block (using a standard unpaired $t$-test) across the three blocks. The existence of genotype (morph) $\times$ environment (diet) interactions for enzyme activities was determined by inspecting the consistency of morph $\times$ environment interactions within blocks across the three blocks.

\section{Results}

\section{Organ and Whole-Body Masses}

On the day of molt to adulthood (day 0), masses of fat bodies, ovaries, and whole bodies were similar between the LW(f) and SW morphs within each block (all $t$-tests were nonsignificant; Table 1). Furthermore, morph means did not differ across blocks (all paired $t$-tests were nonsignificant, Table 1). Similar results were obtained when fat bodies and ovarian masses obtained on day 0 and day 5 (see below) were analyzed by ANCOVA with whole-body mass minus fat body and ovaries as the covariate. By contrast, on day 5 on the high-nutrient (high) diet, large, genetically based differences in fat body and ovarian masses were observed between the morphs. Fat bodies were significantly heavier in LW(f) versus SW morphs within most blocks and across blocks, whereas ovaries were significantly lighter in LW(f) versus SW morphs (Table 1). Mean fat body mass was 33-70\% greater in LW(f) versus SW females, whereas ovarian mass was $135-400 \%$ greater in SW versus LW(f) females. Similar trends were observed on the sucrose and low diets. However, in most cases, differences in fat body and ovarian mass were much smaller between the morphs and did not differ significantly on these diets (Table 1).

\section{Enzyme-Specific Activities on Day of Molt to Adulthood}

In all but three cases (Fas, $A c l$, and G-6-Pdh in block 1), specific activities of fat body enzymes did not differ significantly between LW(f) and SW morph within blocks on the day of molt to adulthood (Table 2). In no case did mean activities differ between morphs across blocks.

\section{Genetically Based Differences in Enzyme Activities between $L W(f)$ and $S W$ Morphs}

One of the most important finding of the present study was the consistently and substantially higher enzyme specific activities in LW(f) versus SW morphs on day 5 of adulthood on the high and sucrose diets (Figs. 2-5). In 33 of 42 cases, specific activity of a particular enzyme was significantly higher in LW(f) versus SW morphs within a block and diet. In six of the nine nonsignificant cases, activities were also higher in LW(f) than in SW females. Finally, mean specific activity was significantly higher (10 of 14 cases) or nearly significantly higher $(0.1>P>0.05$, three of 14 cases $)$ in $L W(f)$ versus SW morphs across blocks as determined by paired $t$-tests. These results indicate large-magnitude, genetically based elevation in specific activities of all studied enzymes in LW(f) versus SW morphs on the high and sucrose diets. The ability to document significant genetic differences between morphs, despite very low statistical power $(d f$ $=2$ ) in the paired $t$-tests, attests to the remarkably large differences between morphs that were consistent across blocks. Averaged over all blocks and enzymes, specific activity was 1.7 fold (70\%) higher in LW(f) than in SW females. The only case where mean enzyme activities did not differ between morphs across blocks on the high and sucrose diets was Me. Nevertheless, even for this enzyme, there was a significant elevation of specific activity in the LW(f) versus the SW morph in each of two blocks and significant cosegregation between Me activity and wing morph (see below).

In contrast to the higher enzyme activities in LW(f) versus SW crickets raised on the high and sucrose diets, much smaller and mainly nonsignificant differences in enzyme activities were observed between morphs on the low diet. Of the seven enzymes studied, paired 


\section{G-6-PDH}
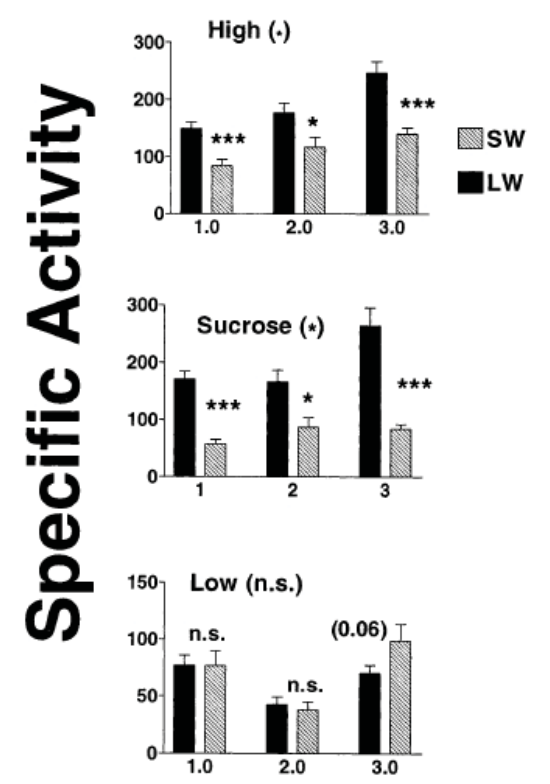

IDH
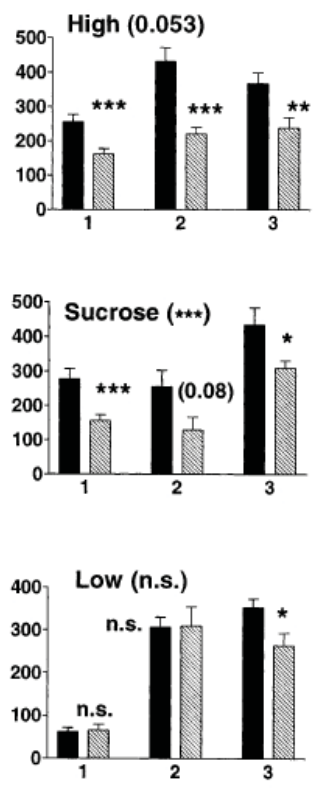

\section{Block}

Figure 2. Mean ( \pm SEM) specific activities of G-6-pdh (glucose-6-phosphate dehydrogenase) and $\mathrm{NADP}^{+}$-dependent $I d h$ (isocitrate dehydrogenase) in LW(f) females from LW-selected lines (black histograms) and SW females from SW-selected lines (hatched histograms) raised on three diets. Numbers on the $x$-axes refer to the block to which the lines belong. Asterisks above histograms refer to results of unpaired $t$-tests comparing specific activities between morphs within a block $(* P<0.05 ; * * P<0.01 ; * * * P<0.005)$. Asterisks within parentheses near diet designations refer to results of paired $t$-tests of differences in mean specific activities between morphs across blocks. In cases where paired or unpaired $t$-tests were nearly significant $(0.1>P>0.05)$, exact $P$-values are given. Activities are in units of $\mathrm{nmol} / \mathrm{min} / \mathrm{mg}$ protein. Sample sizes ranged from five to 13 individuals per mean.

$t$-tests were significant only for $\mathrm{Me}(t=12.49, P=0.006)$ and $\mathrm{Fas}(t$ $=5.31, P=0.04)$. In 15 of 21 cases, morphs did not differ in specific activity for a particular enzyme within a particular block.

Comparisons between morphs discussed above are for specific activities (activity scaled to microgram fat body protein). Relative difference in whole-fat-body enzyme activity between morphs (product of specific activity and fat body mass; see Materials and Methods for calculation; data not shown) was even greater than specific activities on the high and sucrose diets $(P<0.05$ in all paired $t$-tests, except for AspAT on the sucrose diet, $P=0.07)$. This resulted from the significantly larger fat bodies of $\mathrm{LW}(\mathrm{f})$ versus SW females (especially on the high diet; Table 1), which mag-nified the significantly higher specific activities of LW(f) versus SW crickets. Similar to the situation for specific activities on the low diet, relative total-fat-body activities on this diet did not differ between morphs across blocks for five of seven enzymes on that diet (paired $t$-tests, $P>0.1 ;$ Fas, $P=0.03 ; M e, P=0.02$ ).

FAS

ACL
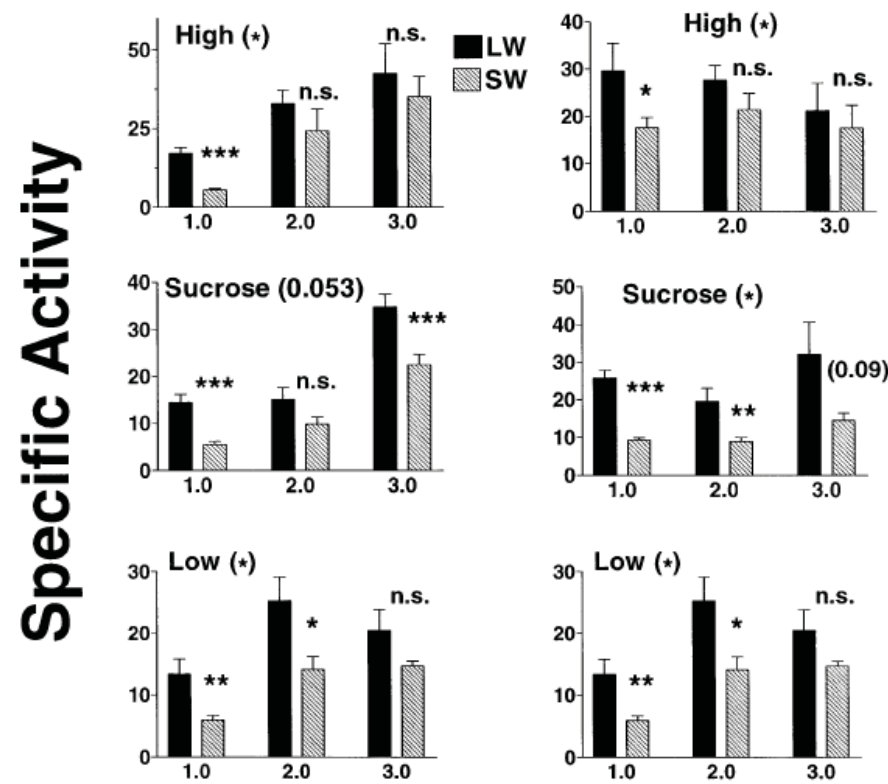

\section{Block}

Figure 3. Mean ( \pm SEM) specific activities of $\mathrm{Fas}$ (fatty-acid synthase) and $\mathrm{Acl}$ (ATP-citrate lyase) in LW(f) females from LW-selected lines (black histograms) and SW females from SW-selected lines (hatched histograms) raised on three diets. See legend of Figure 2 and Materials and Methods for further explanation of symbols. Sample sizes ranged from six to eight individuals per mean.

\section{Genotype $\times$ Environment Interaction for Enzyme Activities}

For three enzymes, Fas, Acl, and AspAT, differences in specific activities between morphs were similar on each of the three diets in each of the three blocks (Figs. 3, 5). For AlaAT a strong morph $(M) \times \operatorname{diet}(D)$ interaction was observed for activities in block 2 (ANOVA, $P=0.001$ ), but not in the other blocks (ANOVA, $P>0.1$; Fig. 5). No consistent pattern of morph-specific differences in enzyme activity as a function of diet was observed across the blocks for this enzyme. At the other extreme, a significant $\mathrm{M} \times \mathrm{D}$ interaction was observed for $G-6-P d h$ activity that was consistent across the three blocks (ANOVA: $P=0.001, P=$ $0.034, P<0.0005$, respectively), providing strong evidence for genotype (morph) $\times$ environment (diet) interaction for $G-6-P d h$ activity (Fig. 2). As can be seen in Fig. 2, this $\mathrm{M} \times \mathrm{D}$ resulted from substantially higher activity in LW(f) versus SW morphs on the high and sucrose diets coupled with no or very minor differences in activities between morphs on the low diet in each of the three blocks. Less strong, but suggestive data indicating a similar $\mathrm{G} \times$ E interaction was observed for the other two NADPH-producing enzymes. For $I d h$ specific activities (Fig. 2), a significant $\mathrm{M} \times \mathrm{D}$ interaction was observed on block $1(P=0.036)$ and block $2(P=$ $0.027)$, but not on block $3(P=0.78)$. Like $G-6-P d h$ activities, a consistent pattern of greater elevation in Idh activity in LW(f) 

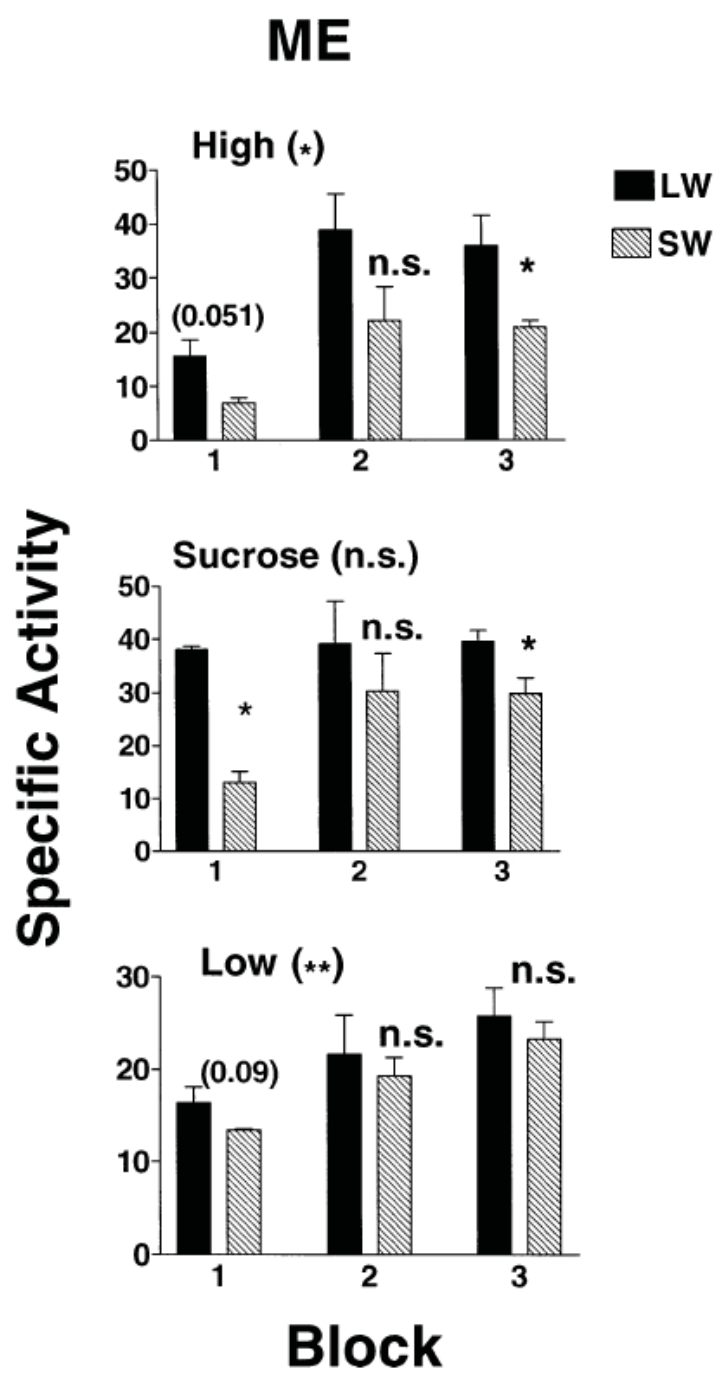

Figure 4. Mean ( \pm SEM) specific activities of $M e$ (malic enzyme) in LW(f) females from LW-selected lines (black histograms) and SW females from SW-selected lines (hatched histograms) raised on three diets. See legend of Figure 2 and Materials and Methods for further explanation of symbols. Sample sizes ranged from three to 10 individuals per mean.

versus SW morphs on the high and sucrose diets was observed in each of the three blocks. Finally, a significant $\mathrm{M} \times \mathrm{D}$ interaction was observed for $M e$ on block $1(P<0.0005)$ but not on the other two blocks $(P>0.1)$, although the differences between the morphs as a function of diet were roughly similar across blocks.

The significantly greater fat body mass in LW(f) versus SW morphs, which occurred primarily on the high diet (Table 1), magnified the $\mathrm{M} \times \mathrm{D}$ interaction for whole-fat-body activities relative to $\mathrm{M} \times \mathrm{D}$ for specific activities. For $M e, G-6-p d h$, and $I d h$, highly significant $(P<0.005) \mathrm{M} \times \mathrm{D}$ interactions were observed for whole-fat-body activity in each block. For Acl, Fas, AspAT, and AlaAT, highly significant $\mathrm{M} \times \mathrm{D}$ interactions were observed in two of three blocks.

\section{Line Crosses}

Specific activities of six enzymes (Fas, Acl, G-6-pdh, Idh, Me, As$p A T)$ were measured in $\mathrm{F}_{2}$ females from each of two backcrosses

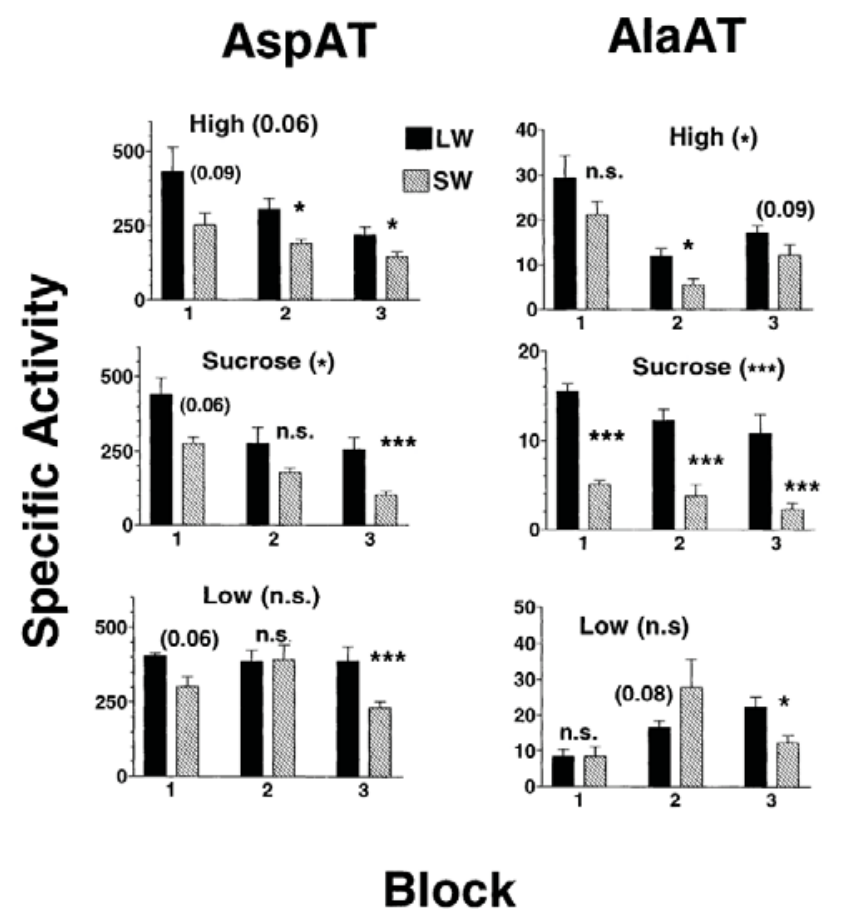

Figure 5. Mean ( \pm SEM) specific activities of AlaAT (alanine aminotransferase) and $A s p A T$ (aspartate aminotransferase) in LW(f) females from LWselected lines (black histograms) and SW females from SW-selected lines (hatched histograms) raised on three diets. See legend of Figure 2 and Materials and Methods for further explanation of symbols. Sample sizes ranged from three to nine individuals per mean.

(crosses 9 and 10; Fig. 6; sample sizes are in the figure legend). Strong cosegregation was observed between all enzyme activities and wing morph (i.e., wing length, flight muscle phenotype, ovarian mass). That is, activity of each of the six enzymes was significantly higher in LW(f) females compared with SW females in $\mathrm{F}_{2}$ females in each of the two crosses (Fig. 6). With one exception, all SW females had small (arrested development) white muscles $(N=22)$, whereas, with one exception, all LW females had large pink (functional) flight muscles (i.e., were LW[f]; $N=$ 22). In a subset of females from cross 9 , all SW females had large ovaries (as percentage body wet mass; median $=13.0 \%$, range $=$ $14.2-9.3 \%, N=7$ ), whereas all LW(f) had small ovaries (median $=2.5 \%$, range $=8.8-2.0 \%, N=7)$. No significant morph $\times$ cross interactions were observed for activities of any enzyme (two-way ANOVAs; $P>0.1$ in all cases) indicating differences of equal magnitude between morphs in the two crosses. In 10 of 12 cases, enzyme-specific activities were 1.5- to 2.1-fold higher in LW(f) versus $\mathrm{SW} \mathrm{F}_{2}$ females (median 1.9), similar to the 1.7 fold $(N=$ 18) median elevation in activity of these six enzymes in LW(f) versus SW females in the parental lines (Figs. 2-5).

\section{Discussion}

Wing polymorphism has recently developed into a powerful experimental model in studies of the evolutionary physiology of life histories and dispersal (Zera and Denno 1997; Zera et al. 1998; Zera and Huang 1999; Zera and Cisper 2001; Zera and Harshman 2001). The present study has expanded this model by providing the first information on the biochemical-genetic cor- 


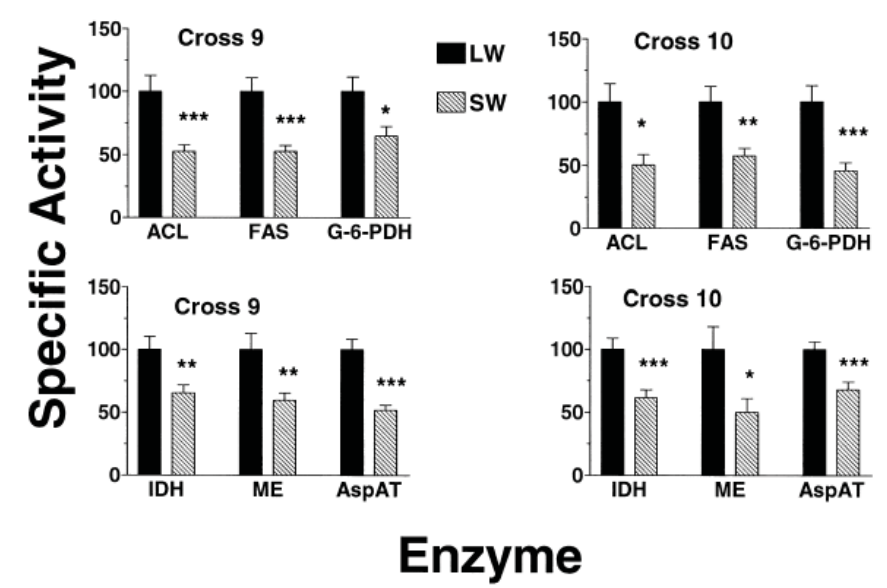

Figure 6. Mean ( \pm SEM) specific activities (nmol/min/mg protein) of six enzymes in $\mathrm{F}_{2}$ backcrossed LW(f) (black histogram) and SW (hatched histogram) female Gryllus firmus. In each of crosses 9 and 10, a SW hybrid female resulting from a single pair cross between the L-1 and S-1 lines was backcrossed to the L-1 or S-1 lines producing the $\mathrm{F}_{2}$ females. Means were based on seven to nine individuals, except for $I d h$ and $M e$ in cross 9, which were based on 11-13 individuals. Activities were scaled such that mean activity in the LW(f) morph equaled 100. Asterisks indicate significant differences in specific activities between LW(f) and SW females at $* P<0.05 ; * * P<0.01$; or $* * * P<0.005$. See the legend for Figure 2 for additional explanation of symbols.

relates of morph specialization for reproduction and dispersal. Three noteworthy results were obtained that will be discussed in more detail below. First, morphs of G. firmus that differ genetically in life-history and dispersal traits also differ genetically in the activities of each of six enzymes of lipid and amino acid metabolism (Figs. 2-5). Second, cosegregational analysis demonstrated strong genetic associations among enzyme activities, morphological aspects of flight capability (wing length and flight muscle mass), and reproductive traits (i.e., ovarian growth; Results, Fig. 6). These results, together with additional data (discussed below), strongly imply that differences between morphs in the activities of enzymes of lipid and amino acid metabolism are important functional components of morph-specific adaptations for enhanced early reproduction versus dispersal capability. The strong cosegregation of morphological, physiological, and biochemical traits in G. firmus also has important implications regarding the proximate mechanisms coordinating the expression of traits that constitute components of morph specialization and the evolution of these regulatory mechanisms. Finally, dramatic genotype (morph) $\times$ environment (diet) interactions were observed for activities of many enzymes (Results), indicating that activity differences between morphs are often strongly contingent upon the diet.

\section{Functional Significance of Enzyme Activity Differences between Morphs}

Zera and Larsen (2001) previously documented that the LW(f) morph of G. firmus has a much higher (38\%; mass-adjusted) triglyceride content than the SW morph. The elevated content of triglyceride, which is the most important flight fuel in species of Gryllus (Zera et al. 1999), was of large magnitude, genetically based, and produced during the first week of adult- hood. The elevated triglyceride content also was strongly associated with substantially reduced (50-75\%) ovarian growth in the LW(f) morph and was not due to enhanced lipid assimilation from the diet (Zera and Brink 2000). These data were collectively viewed as strongly supporting the hypothesis that there is a direct trade-off between internal nutrients allocated to ovarian growth versus flight fuel (triglyceride) production in morphs of G. firmus during the first week of adulthood (Zera and Harshman 2001; Zhao and Zera 2001). Results of the present study provide both additional support for this hypothesis and information on the mechanisms underlying adaptive morph differentiation in triglyceride production.

The present study focused on a diverse set of enzymes that were chosen because they occupy strategic points in pathways of lipid metabolism or link pathways of amino acid and lipid metabolism (Downer 1985; Fig. 1). For example, fatty acid synthase (Fas) exists within the main pathway of fatty acid biosynthesis, whereas $\mathrm{Acl}$ is involved in production of acetyl CoA, a key precursor of lipid biosynthesis. Three enzymes, G-6$p d h, M e$, and $N A D P^{+}-I d h$, exist in various pathways involved in the production of NADPH, a cofactor required for reductive biosynthesis of fatty acids. Finally, alanine aminotransferase (AlaAT) and aspartate aminotransferase (AspAT) convert amino acids into Krebs cycle intermediates that have a variety of metabolic fates, one of which is conversion into lipid. Many studies have documented the importance of Fas, Acl, G-6-pdh, Me, and $N A D P^{+}$-Idh in lipid biosynthesis (reviewed in Downer 1985). Activities of these enzymes are typically elevated during periods of lipogenesis in insects (Downer 1985) and are higher in selected lines of insects and mammals that contain elevated lipid levels (e.g., Asante et al. 1989; Clark et al. 1990; Hastings and Hill 1990; Harshman et al. 1999).

Developmental profiles and morph-specific differences in activities of Fas, Acl, G-6-pdh, Me, and NADP ${ }^{+}-I d h$ parallel previously reported morph-specific patterns of triglyceride accumulation in G. firmus. At the adult molt (day 0), morphs have low and equivalent activities of biosynthetic enzymes (Table 2) and triglyceride content (Zera and Larsen 2001). By day five of adulthood, activities of each of these five enzymes and triglyceride content are significantly higher in LW(f) versus SW females on the high and sucrose diets (Figs. 2-5; Zera and Larsen 2001). Remarkably, every enzyme in lipid metabolism that was studied was altered to roughly the same degree between morphs. These parallel patterns strongly suggest that the elevated triglyceride content in the LW(f) morph results from large-scale alterations in the activities of many enzymes of lipid metabolism leading to an increased biosynthesis of triglyceride.

Because of the complexity of intermediary metabolism, it is very risky to conclude that enzyme activity differences between genotypes, measured in vitro, are the cause of differences between genotypes in the end products of in vivo metabolism (e.g., triglyceride), unless information is available on genotype-dependent flux through metabolic pathways measured in vivo (Zera et al. 1985; Dykhuizen and Dean 1990; Zera and Huang 1999). It is therefore noteworthy that we have directly measured large-scale phenotypic (Zhao and Zera 2001) and genetic (Zhao and Zera 2002) differences between morphs in rates of lipid biosynthesis using radio-labeled fatty acid and fatty acid precursors. These 
differences in lipid biosynthesis were measured in vivo and parallel differences in enzyme activities between the morphs measured in vitro. That is, rates of lipid biosynthesis were low and equivalent in the morphs on day 0 of adulthood, but were significantly higher in LW(f) than in SW females on day 5 of adulthood on the high and sucrose diets. Additional radiotracer studies also have documented consistently greater conversion of amino acid into lipid in LW(f) versus SW morphs in each of the three blocks (Z. Zhao and A. J. Zera, unpublished data). Thus, the higher in vitro activities of the two aminotransferases, AlaAT and AspAT, in the LW(f) morph (Fig. 5), appear to reflect higher in vivo rates of conversion of amino acids into lipid in that morph. Studies of aminotransferases are especially important in understanding the biochemical basis of life-history trade-offs because they provide information on the mechanisms linking pathways of amino acid metabolism, which are important in yolk protein production (i.e., reproductive effort), with pathways of lipid metabolism, which are important in somatic maintenance.

On the high diet, and to a lesser degree, on the sucrose diet, total-fat-body enzyme activities (activity $\times$ organ mass) were elevated to a greater degree than specific activities (i.e., activities scaled to organ mass) in LW(f) versus SW females (Results). Indeed, on the high diet, the $50 \%$ elevation in fat body mass in LW(f) versus SW females was nearly as great as the $70 \%$ higher specific activities of fat body enzymes of the LW(f) morph. Thus, it appears that evolutionary modification of mass of the fat bodies plays nearly as important a role as modification of the metabolic properties of fat bodies in producing elevated rates of lipid biosynthesis in LW(f) females. Although enzyme activities have been compared between lines that differ in life-history traits in a number of studies of Drosophila (e.g., Harshman and Schmidt 1998; Harshman et al. 1999), the present study is the first in insects to document the relative importance of variation in specific activities and organ mass to differences in whole-organism activity between life-history phenotypes. Activities of enzymes often differ dramatically among organs (Newsholme and Start 1973). Thus, understanding the biochemical causes of life-history variation requires that biochemical traits be measured in individual organs of life-history phenotypes rather than in homogenates of whole organisms as has typically been done (e.g., in virtually all Drosophila studies).

Variation in fat body mass between morphs likely results from either space or hormonal constraints. In the SW morph the much larger ovaries, in and of themselves, may necessitate reduced fat body mass because of limited abdominal volume. In the congener G. assimilis, hormonal manipulation produced a strong negative correlation between ovarian and gut masses that was thought to be due to a size constraint of the abdomen (Zera et al. 1998). Alternatively, long-duration elevation of the juvenile hormone $(\mathrm{JH})$ titer in the SW morph (Zera and Cisper 2001), which may be necessary for enhanced ovarian growth in that morph, may reduce fat body mass, similar to the known effect of JH on flight muscle degeneration (Zera and Cisper 2001). In the present study, fat body masses also were considerably lower in both morphs on the low diet (Table 1), probably due to reduced nutrients in the low diet (75\% less nutrients than the high diet), which limited organ growth. LW(f) females lose mass and SW females barely gain mass when fed the low diet (A. J. Zera, unpublished data). The lower activities of lipogenic enzymes on the low diet probably reflect an overall reduction in biosynthesis on that diet. Finally, variation in organ mass between the low and the high and sucrose diets was also a major contributor to the significant genotype $\times$ environment interaction for many whole-fat-body enzyme activities (Results). LW(f) and SW morphs differed in both enzyme activity and fat body mass on the high and sucrose diets, whereas both enzyme activities and fat body mass were much similar between the morphs on the low diet.

Although lipid biosynthesis has been discussed above in the context of flight fuel production, lipid is also a major component of eggs of Gryllus (Zera et al. 1984) and other insects (Downer 1985). Thus, lipid biosynthesis is also of critical importance for the elevated egg production in the flightless SW morph. This is evidenced by the increase in activities of lipogenic enzymes (Table 2, Figs. 2-5) and rate of fatty acid biosynthesis (Zhao and Zera 2001) during the first five days of adulthood in the SW morph, when substantial ovarian growth is occurring. The important point with respect to the tradeoff between reproduction and flight capability is that reduced egg production in the LW(f) morph allows an increased amount of nutrients to be allocated to flight fuel (triglyceride) biosynthesis (present study; Zera and Larsen 2001; Zhao and Zera 2001). For example, recent radiotracer studies have shown that the LW(f) morph converts a greater amount of amino acids to lipid precursors, which are then converted into lipid, in contrast to the SW morph, which allocates a greater amount of amino acids to production of egg proteins (A. J. Zera and Z. Zhao, unpublished data). Recent radiotracer studies also indicate that morph-specific differences in lipid biosynthesis are more complex than simple quantitative differences in the amount of total lipid produced. Morphs differ in both the relative biosynthesis of various lipid classes that are important in flight versus egg production and the organs to which these biosynthesized lipids are allocated (e.g., ovary vs. fat bodies; Zhao and Zera 2002).

\section{Causes of Coordinate Activity Differences between Morphs}

Another important finding of the present study was the strong cosegregation among activities of all studied enzymes, morphological aspects of flight capability, and reproductive traits in $G$. firmus (Results, Fig. 6). $\mathrm{F}_{2}$ backcrossed females that had long wings also had large functional flight muscles, small ovaries, and high activities of each of six enzymes. When activities of all enzymes were considered, the magnitude of elevation in specific activity in $\mathrm{F}_{2} \mathrm{LW}(\mathrm{f})$ versus $\mathrm{SW}$ females (median elevation $=1.9$ fold; i.e., $90 \%$ higher) was similar to that between morphs from the parental lines (median elevation $=1.7$ fold; $70 \%$ higher). These results clearly show that the various morphological, physiological, and biochemical traits that vary between genetically specified LW(f) and SW morphs strongly cosegregate as a single unit. We cannot discount linkage as the cause of these strong genetic correlations. However, a more likely explanation is that some master regulatory factor(s) are genetically polymorphic in G. firmus, resulting in the coordinate expression of the various morphological, reproductive, and enzymatic traits that define flight-capable and flightless morphs. An obvious candidate regulator is juvenile hormone $(\mathrm{JH})$, which is known to reduce the expression of wing length, flight muscle mass, and triglyceride content and to enhance ovarian growth in several species of Gryllus and other insects (Zera and Tiebel 1988; Zera and Tanaka 1996; Zera et al. 1998; Zera and Cisper 2001 and 
references therein). Indeed, recent studies have shown that $\mathrm{JH}$ applied to LW(f) females causes a reduction in enzyme activities and rates of lipid biosynthesis to levels found in the SW morph (i.e., produces a SW biochemical phenocopy; Z. Zhao and A. J. Zera, unpublished data). JH titers are known to differ between morphs of G. firmus (Zera and Cisper 2001). Thus, one possible cause of the coevolutionary changes in metabolism, reproduction, and morphology in G. firmus is the evolutionary modification of regulatory factors such as $\mathrm{JH}$, which directly or indirectly influence the expression of each of these traits. Recent chemostat selection studies in the yeast Saccharomyces cerevisiae (Ferea et al. 1999) provide support for the hypothesis that evolutionary alterations of many enzymes in metabolism can result from selection on a limited number of variable regulators. Selection for growth on glucose-limited media for only several hundred generations resulted in independently evolved strains of $S$. cerevisiae with elevated growth rates. DNA microarray studies documented that these strains all differed from the ancestral clone from which they were derived in the expression of hundreds of loci, many of which encode enzymes of central metabolism such as glycolysis, the Krebs cycle, oxidative phosphorylation, and electron transport. Ferea et al. (1999) argued that selection on a small number of mutants that regulate the expression of functionally related loci was the most likely explanation for the rapid evolution of these strains. A key unresolved issue in life-history physiology is the extent to which evolutionary modification of regulatory controls underlies the evolution of new life-history traits (Zera and Harshman 2001). Wing polymorphism in G. firmus may be an excellent experimental model to approach this problem, given the very large differences in life-history and metabolic traits and the extensive background information on endocrine variation between morphs (Zera and Huang 1999; Zera and Cisper 2001; Zera and Harshman 2001).

\section{Future Prospects: Integrating Evolutionary Studies of Life Histories and Metabolic Biochemistry}

To a large extent, variation in life-history traits is determined by variation in aspects of metabolism involved in the production of life-history components, such as yolk protein for eggs, triglyceride for somatic maintenance and dispersal, and energy for growth. Thus, a deep understanding of the mechanisms underlying lifehistory evolution requires detailed knowledge of the aspects of intermediary metabolism that have been altered to produce phenotypic variation and covariation in these life-history components. Only by opening the black box of intermediary metabolism can the chain of causality be traced from variation in DNA sequence to variation in the phenotypic expression of whole-organism life histories. Detailed information on variation within and interactions among metabolic pathways will be required to understand many central issues in life-history evolution, such as the nature and stability of genetic correlations among life-history traits and the nature of constraints in life-history evolution (for a recent excellent example of this latter point, see O'Brien et al. 2002).

Thus far, the synthesis between life-history evolution and metabolic biochemistry is at a rudimentary stage. For example, despite the considerable number of inter- and intraspecific studies of biochemical adaptation of metabolic pathways and enzymes
(Hochachka and Somero 2002; Watt 1985; Zera et al. 1985; Prosser 1986; Powers 1987; Clark 1989, 1990), only a handful of investigations have explicitly focused on variation in activities of enzymes of central metabolism in the context of life-history microevolution (e.g., present study; Harshman and Schmidt 1998; Harshman et al. 1999; Zhao and Zera 2001; reviewed in Zera and Harshman 2001). Even fewer studies have quantified in vivo differences in flux through metabolic pathways in organisms that differ in life-history traits (Boggs 1997; Zhao and Zera 2001), and no comparable metabolic-genetic studies have been reported except for Zhao and Zera (2002). Finally, evolutionary modification of the regulatory controls of intermediary metabolism in the context of life-history evolution remains virtually unstudied. The present study, in which large-magnitude differences in the biochemical-genetic correlates of life-history variation and trade-offs were documented, attests to the utility of wing polymorphism as a model for integrating studies of life-history evolution with metabolic biochemistry.

\section{Acknowledgments}

Research reported here was supported by National Science Foundation grant IBN 9808249.

\section{Literature Cited}

Asante E. A., W. G. Hill, G. Bulfield. 1989. Analysis of lines of mice selected for fat content. 1. Correlated responses in the activities of NADPH-generating enzymes. Genet. Res. Camb. 54:155160.

Boggs C. 1997. Dynamics of reproductive allocation from juvenile and adult feeding: radiotracer studies. Ecology. 74:433-441.

Chippindale A. K., T. J. Chu, M. R. Rose. 1996. Complex trade-offs and the evolution of starvation resistance in Drosophila melanogaster. Evolution. 50:753-766.

Clark A. G. 1989. Causes and consequences of variation in energy storage in Drosophila melanogaster. Genetics. 123:131-144.

Clark A. G. 1990. Genetic components of variation in energy storage in Drosophila melanogaster. Evolution. 44:637-650.

Clark A. G., F. M. Szumski, K. Bell, L. E. Keith, S. Houtz, D. A. Merriwether. 1990. Direct and correlated responses to artificial selection on lipid and glycogen contents in Drosophila melanogaster. Genet. Res. Camb. 56:49-56.

Djawdan M., T. T. Sugiyama, L. K. Schlaeger, T. J. Bradley, M. R. Rose. 1996. Metabolic aspects of the trade-off between fecundity and longevity in Drosophila melanogaster. Physiol. Zool. 69:1176-1195.

Downer R. G. H. 1985. Lipid metabolism. Pp. 77-114 in G. A. Kerkut and L. I. Gilbert, eds. Comprehensive insect physiology, biochemistry and pharmacology. Pergamon, Oxford, U.K.

Dykhuizen D. E., A. M. Dean. 1990. Enzyme activity and fitness: evolution in solution. Trends Ecol. Evol. 5:257-262.

Ferea T. L., D. Botstein, P. O. Brown, R. F. Rosenzweig. 1999. Systematic changes in gene expression patterns following adaptive evolution in yeast. Proc. Natl. Acad. Sci. USA. 96:9721-9726.

Fisher R. A. 1930. The genetical theory of natural selection. Dover, New York.

Geer B. W., C. C. Laurie-Ahlberg. 1984. Genetic variation in the dietary sucrose modulation of enzyme activities in Drosophila me- 
lanogaster. Genet. Res. Camb. 43:307-321.

Harshman L. G., A. A. Hoffmann. 2000. Laboratory selection experiments using Drosophila: What do they really tell us? Trends Ecol. Evol. 15:32-36.

Harshman L. G., J. L. Schmidt. 1998. Evolution of starvation resistance in Drosophila melanogaster: aspects of metabolism and counter-impact selection. Evolution. 52:1679-1685.

Harshman L. G., A. A. Hoffmann, A. G. Clark. 1999. Selection for starvation resistance in Drosophila melanogaster: physiological correlates, enzyme activities and multiple stress responses. J. Evol. Biol. 12:370-379.

Hastings I. M., W. G. Hill. 1990. Analysis of lines of mice selected for fat content: correlated responses in the activities of enzymes involved in lipogenesis. Genet. Res. Camb. 55:55-61.

Hochachka P. W., G. N. Somero. 2002. Biochemical adaptation. Oxford Univ. Press, New York.

Mole S., A. J. Zera. 1993. Differential allocation of resources underlies the dispersal-reproduction trade-off in the wing-dimorphic cricket, Gryllus rubens. Oecologia. 93:121-127.

Newsholme E. A., C. Start. 1973. Regulation in metabolism. John Wiley and Sons, London.

O’Brien D. M., M. L. Marilyn, C. Boggs. 2002. Renewable and nonrenewable resources: amino acid turnover and allocation to reproduction. Proc. Natl. Acad. Sci. USA. 99:4413-4418.

Pianka E. R. 1981. Resource acquisition and allocation among animals. Blackwell Scientific Publications, Oxford, U.K.

Powers D. A. 1987. A multidisciplinary approach to the study of genetic variation within species. Pp. 102-134 in M. E. Feder, A. F. Bennett, W. W. Burggren, and R. B. Huey, eds. New directions in ecological physiology. Cambridge Univ. Press, Cambridge, U.K.

Prosser C. L. 1986. Adaptational biology: molecules to organisms. John Wiley and Sons, New York.

Rose M. R., T. J. Nusbaum, A. K. Chippindale. 1996. Laboratory evolution: the experimental wonderland and the Cheshire cat syndrome. Pp. 221-241 in M. R. Rose and G.V. Lauder, eds. Adaptation. Academic Press, San Diego, CA.

Service P. M. 1987. Physiological mechanisms of increased stress resistance in Drosophila melanogaster selected for postponed senescence. Physiol. Zool. 60:321-326.

Stoscheck C. M. 1990. Quantitation of protein. Meth. Enzymol. 182:60-63.

Townsend C. R., P. Calow. 1981. Physiological ecology: an evolutionary approach to resource use. Blackwell Scientific Publications, Oxford, U.K.

Veazy J. N., C. A. R. Kay, T. J. Walker, W. H. Whitcomb. 1976. Seasonal abundance, sex ratio, and macroptery of field crickets in northern Florida. Ann. Entomol. Soc. Am. 69:374-380.

Watt W. B. 1985. Bioenergetics and evolutionary genetics: opportunities for new synthesis. Am. Nat. 125:118-143.

Zera A. J., J. Bottsford. 2001. The endocrine-genetic basis of lifehistory variation: relationship between the ecdysteroid titer and morph-specific reproduction in the wing-polymorphic cricket, Gryllus firmus. Evolution. 55:538-549.
Zera A. J., T. Brink. 2000. Nutrient absorption and utilization by wing and flight muscle morphs of the cricket Gryllus firmus: implications for the trade-off between flight capability and early reproduction. J. Insect Physiol. 46:1207-1218.

Zera A. J., G. C. Cisper. 2001. Genetic and diurnal variation in the juvenile hormone titer in a wing-polymorphic cricket: implications for the evolution of life histories and dispersal. Physiol. Biochem. Zool. 74:293-306.

Zera A. J., R. F. Denno. 1997. Physiology and ecology of dispersal polymorphism in insects. Annu. Rev. Entomol. 42:207-231.

Zera A. J., L. G. Harshman. 2001. The physiology of life-history trade-offs in animals. Annu. Rev. Ecol. Syst. 32:95-126.

Zera A. J., Y. Huang. 1999. Evolutionary endocrinology of juvenile hormone esterase: functional relationship with wing polymorphism in the cricket, Gryllus firmus. Evolution. 53:837-847.

Zera A. J., A. Larsen. 2001. The metabolic basis of life-history variation: genetic and phenotypic differences in lipid reserves among life-history morphs of the wing-polymorphic cricket, Gryllus firmus. J. Insect Physiol. 47:1147-1160.

Zera A. J., S. Tanaka. 1996. The role of juvenile hormone and juvenile hormone esterase in wing morph determination in Modicogryllus confirmatus. J. Insect Physiol. 42:909-915.

Zera A. J., K. C. Tiebel. 1988. Brachypterizing effect of group rearing, juvenile hormone-III, and methoprene on wing-length development in the wing-dimorphic cricket, Gryllus rubens. J. Insect Physiol. 34:489-498.

Zera A. J., S. Mole, K. Rokke. 1984. Lipid, carbohydrate and nitrogen content of long-and short-winged Gryllus firmus: implications for the physiological cost of flight capability. J. Insect Physiol. 40:1037-1044.

Zera A. J., R. K. Koehn, J. G. Hall. 1985. Allozymes and biochemical adaptation. Pp. 633-674 in G. A. Kerkut and L. I. Gilbert, eds. Comparative insect physiology, biochemistry, and pharmacology. Vol. 10. Pergamon Press, Oxford, U.K.

Zera A. J., J. Sall, K. Grudzinski. 1997. Flight-muscle polymorphism in the cricket Gryllus firmus: muscle characteristics and their influence on the evolution of flightlessness. Physiol. Zool. 70:519529.

Zera A. J., J. Potts, K. Kobus. 1998. The physiology of life-history trade-offs: experimental analysis of a hormonally induced lifehistory trade-off in Gryllus assimilis. Am. Nat. 152:7-23.

Zera A. J., J. Sall, K. Otto. 1999. Biochemical aspects of flight and flightlessness in Gryllus: flight fuels, enzyme activities, and electrophoretic profiles of flight muscles from flight-capable and flightless morphs. J. Insect Physiol. 45:275-285.

Zhao Z., A. J. Zera. 2001. Enzymological and radiotracer studies of lipid metabolism in the flight-capable and flightless morphs of the wing-polymorphic cricket, Gryllus firmus. J. Insect Physiol. 47:1337-1347.

Zhao Z. 2002. Differential lipid biosynthesis underlies a trade-off between reproduction and flight capability in a wing-polymorphic cricket. Proc. Natl. Acad. Sci. USA. 99:16829-16834. 